A. John Sommese

Nagoya Math. J.

Vol. 86 (1982), 101-129

\title{
COMPLEX SUBSPACES OF HOMOGENEOUS COMPLEX MANIFOLDS II-HOMOTOPY RESULTS
}

\author{
ANDREW JOHN SOMMESE \\ To the memory of my brother Joseph
}

The Lefschetz hyperplane section theorem has roots going back at least to Picard, but it was Lefschetz [20] who first stated and proved it in the modern form for integer homology. Later it was improved up to the homotopy level by Andreotti-Frankel [1] and Bott [8] using an idea of Thom. Numerous generalizations along the same lines have appeared, e.g. [14, Theorem H], [19], [24, App. II] etc.

In [4] Barth proved an unexpected generalization of Lefschetz's theorem for the complex cohomology of arbitrary complex submanifolds of $\boldsymbol{P}_{C}^{N}$.

Hartshorne [15, 16], Ogus [23], and more recently Hartshorne-Speiser [17] have algebraicized these results for projective spaces even over other fields. Barth and Larsen [cf. 5] improved Barth's theorem for A, a submanifold of $\boldsymbol{P}_{C}^{N}$, to the level of homotopy:

$$
\pi_{j}\left(\boldsymbol{P}_{\boldsymbol{C}}^{N}, A, x\right)=0 \quad \text { for } x \in A \text { and } j \leq 2 \operatorname{dim}_{C} A-N+1 .
$$

This paper is devoted to generalizations of the Barth-Larsen results to arbitrary not necessarily compact homogeneous complex manifolds.

One special corollary [cf. §3] that gives the flavour of my results is:

Corollary. Let $B$ and $A$ be complex submanifolds of a homogeneous connected complex manifold $X$ where $A$ is assumed connected. There is an integer $\lambda$ such that:

$\pi_{j}(A, A \cap B, x)=0 \quad$ for $x \in A \cap B$ and $j \leq \min \left\{\operatorname{dim}_{c} A, \operatorname{dim}_{C} B+1\right\}-\lambda$

a) If $X$ is a simple Abelian variety (i.e., $X$ has no sub Abelian varieties), then $\lambda$ can be taken to be $\operatorname{cod}_{c} B$.

b) If $X$ is $\prod_{i=1}^{n} \operatorname{Gr}\left(r_{i}, n_{i}\right)$ where $\operatorname{Gr}\left(r_{i}, n_{i}\right)$ is the Grassmannian of $\boldsymbol{P}_{c}^{r_{i}}$ 's in $\boldsymbol{P}_{c}^{n_{i}}$, then $\lambda$ can be taken to be $\operatorname{cod}_{c} B-\min _{i}\left\{n_{i}\right\}+\operatorname{dim}_{C} X$,

c) If $B$ is compact and has an ample normal bundle then $\lambda$ can be Received November 19, 1979. 
taken to be $\operatorname{cod}_{C} B$ where $X$ and $A$ are not necessarily compact.

The plan of the paper and the proof goes as follows.

In $\S 0$ I give a variant of the basic result of Morse theory on complex manifolds in the form I need it. In $\S 1 \mathrm{I}$ prove a parametrized variant of this result. It says roughly that if $A_{t}$ is a continuous family of complex submanifolds of a complex manifold $X$, and if $B$ is a complex subspace with a 'suitably concave' neighborhood, then the homotopy groups $\pi_{j}\left(A_{t}, A_{t} \cap B, x_{t}\right)$ with $x_{t} \in A_{t} \cap B$, are independent of $t$ in a certain range of dimensions, $j$.

Results in $\S 1$ lead to maps $f: X^{\prime} \rightarrow Y^{\prime}$ which resemble fibrations up to a certain dimension. In $\S 2$, which is mainly topology, I prove the existence of a long exact homotopy sequence for these $f$ up to the appropriate dimension. The types of maps I study are reminiscent of the "quasi-faserungen" of Dold-Thom [10]. Unfortunately, my maps are not as well behaved as their maps, and this causes difficulties. The hardest part of the proof is, as expected, dealing with the difficulties entailed by the fundamental groups. As a trivial consequence we show (2.4) that $\boldsymbol{P}_{\boldsymbol{C}}^{N+1}-A^{\prime}$ cannot be $\lambda$ convex for $\lambda \leq N-2$ where $A^{\prime}$ is the cone on a projective submanifold $A$ of $\boldsymbol{P}_{C}^{N}$ with non-zero first Betti number.

In $\S 3, I$ use the machinery of $\S 1$ and $\S 2$ to give an 'easy proof' of the 'generalized Lefschetz theorem' [cf. the above corollary]. I refer to [25] where I discuss the argument used in $\S 3$ in the case of a simple Abelian variety. I recommend that the reader keep this case of $X$, a simple Abelian variety, in mind. This is conceptually the simplest case because isotropy groups do not obscure matters, but technically the most difficult case for fundamental group reasons.

In $\S 4$, I discuss some open problems and give some examples.

I would like to thank I. Berstein, W. Dwyer, L. Kaup, and the late H. C. Wang for helpful comments and discussions. I am especially grateful to N. Goldstein for a list of corrections that helped in revising this paper. I would also like to thank the NSF and Sonderforschungsbereich 'Theoretische Mathematik' der Universität Bonn for their support.

\section{§. Background material}

In this section I will recall the basic facts about Morse theory on complex manifolds. These results are standard, but not usually stated in the form that I need. 
(0.1) Given a set $S$ in a topological space $X$, let $\bar{S}$ denote the closure of $S$ in $X$.

The following is standard.

(0.2.1) Lemma. Let $A$ be a compact analytic subset of $X$, a connected complex manifold. Let $h: X \rightarrow \boldsymbol{R}$ be a continuous bounded non-negative function on $X$ with $h^{-1}(0)=A$. Assume that $h$ is $C^{\infty}$ on $X-A$. Then given any compact set $K \subseteq X-A$, any $\varepsilon>0$, and any open set $V \supseteq A$, one can choose a $C^{\infty}$ function $H$ on $X$ such that:

a) $H$ is a Morse function on $(X-V) \cup K$

b) $\sup _{x}|H-h|<\varepsilon$,

c) $H$ is as close as one wants to $h$ in the uniform $C^{2}$ topology on $K$.

In particular if the Levi form of $h$ has at most $q-1$ non-negative eigenvalues at each point of $K$, then the same can be assumed of $H$.

Proof. First [22, p. 37] one can choose $C^{\infty}$ Morse function $\tilde{H}$ on $X$ $-A$ that satisfies c) and:

$$
\sup _{x \rightarrow A}|\tilde{H}-h|<\frac{\varepsilon}{2}
$$

Let $\rho$ be a $C^{\infty}$ non negative function bounded by 1 on $X$, that is 0 in some neighborhood of $A$ and 1 on $X-h^{-1}([0, \delta])$, where $\delta$ is chosen $<\varepsilon / 2$ and small enough so that $h^{-1}([0, \delta]) \subseteq V-K \cap V$.

Now let $H=\tilde{H} \rho$.

$H$ is a Morse function on $(X-V) \cup K$ since $\tilde{H}$ is a Morse function on $X-A$ and $H=\tilde{H}$ on $X-h^{-1}([0, \delta]) \supseteq X-(V-V \cap K)=(X-V)$ $\cup K$.

Next note:

$$
\sup _{X}|H-h|=\sup _{X-A}|\tilde{H} \rho-h| \leq \sup _{X-A}|(\tilde{H}-h) \rho|+\sup _{X-A}|h-h \rho| \leq \delta+\frac{\varepsilon}{2}<\varepsilon .
$$

Finally note that $\tilde{H}=H$ on $K$.

The last assertion about the Levi form follows by compactness, since the condition of a Levi form of a function having a certain number of negative eigenvalues is an open condition in the $C^{2}$ topology.

The following notation will be helpful. Given a real valued function, $h$, on a set $X$, let $E(h, \alpha, \beta)=h^{-1}((\alpha, \beta))$, and let $E(h, \alpha)=h^{-1}((-\infty, \alpha))$.

(0.2.2) Lemma. Let $g$ and $F$ be two real valued functions on a set $X$. Assume $\sup _{X}|g-F|<\varepsilon$. Then for all $c \in R$, one has: 


$$
E(F, c) \subseteq E\left(g, c+\varepsilon^{\prime}\right) \subseteq E\left(F, c+\varepsilon^{\prime \prime}\right)
$$

for all $\varepsilon^{\prime} \geq \varepsilon$ and all $\varepsilon^{\prime \prime} \geq \varepsilon^{\prime}+\varepsilon$.

Proof. Trivial.

(0.3) Letting $f$ be a $C^{\infty}$ real valued function on complex manifold $X$, I follow [12; p. 258] and define:

$$
H_{x}(f)=\left\{\left.\xi \in T_{X}\right|_{x}|\partial f|_{x}(\xi)=0\right\}
$$

where $\left.T_{X}\right|_{x}$ is the holomorphic tangent space to $X$ at $x \in X$.

Recall that a $C^{\infty}$ real valued function $f$ on a complex manifold $X$ is called $q$ convex if at each point $x \in X$, the Levi form of $f$ has at most $q-1$ non-positive eigenvalues on $\left.T_{X}\right|_{x}$.

(0.3.1) Definition. Let $A$ be an analytic subset of a connected complex manifold, $X$. An asymptotically $q$ convex exhaustion $f$ of $X-A$ is a $C^{\infty}$ real valued function $f$ on $X-A$ such that:

(0.3.1.1) given any sequence $\left\{x_{n}\right\} \subseteq X-A$ with $x_{n}$ converging to $a \in A$, then $\lim _{n \rightarrow \infty} f\left(x_{n}\right)=\infty$,

and,

(0.3.1.2) the Levi form of $f$ restricted to $H_{x}(f)$ has at most $q-1$ nonpositive eigenvalues where $x \in X-A$.

(0.3.2) Let $A, X$, and $f$ be as in (0.3.1). If $A$ is also compact, then it follows that there is a neighborhood $U$ of $A$ and a $C \in R$ such that $\left(\left.f\right|_{U-A}\right)-C$ is a proper map from $U-A$ to $(0, \infty)$. This is easy to see, e.g. [28, (0.3.3)].

(0.4) Proposition. Let $A$ be a compact analytic subset of a connected complex manifold $X$. Let $f: X-A \rightarrow(0, \infty)$ be a proper map which is an asymptotically $q$ convex exhaustion of $X-A$. Let $X_{\alpha}=\{x \in X-A \mid f(x)$ $>\alpha\} \cup A$. Given a set $\left\{c_{j} \mid 1 \leq j \leq k\right\} \subseteq \boldsymbol{R}$ with $0<c_{1}<\cdots<c_{k}$, there exist open sets $\left\{B_{j} \mid 1 \leq j \leq k-1\right\}$ with smooth boundaries such that:

$$
X_{c_{1}} \supseteq B_{1} \supseteq X_{c_{2}} \supseteq \cdots \supseteq X_{c_{k}}
$$

and,

(0.4.2) given any $x \in A, \pi_{i}\left(B_{j}, A, x\right)=0$ for $i \leq \operatorname{dim}_{C} X-q$ and all $j$.

Proof. Note that $e^{-f}$ is $C^{\infty}$ on $X-A$ and extends to a continuous 
function $g$ on $X$ with $g^{-1}(0)=A$. It is clear that (0.4.1) and (0.4.2) will follow if it is shown that (in the notation of (0.2.2)):

(0.4.3) given any $\{c, d\} \subseteq R$, with $0<c<d<1$, then there is an open set $B$ of $X$ with a smooth boundary such that $E(g, c) \subseteq B \subseteq E(g, d)$ and $\pi_{i}(B, A, x)=0$ for any $x \in A$ and all $i \leq \operatorname{dim}_{C} X-q$.

Choose a neighborhood $W$ of $A$ in $E(g, c)$ such that $A$ is a strong deformation retract of $W$. This is possible since $X$ can be triangulated so that $A$ is a sub-simplicial complex of $X$, and since $E(g, c) \supseteq A$ is open in $X$.

Since $g$ is continuous, there exists an $\alpha>0$ such that $E(g, \alpha) \subseteq W$. Let $K=\overline{E\left(g, \alpha^{\prime}, d\right)}$ where $0<\alpha^{\prime}<\alpha$.

(0.4.4) Let $F=1-e^{-c g}$ where the real number $C>0$ has been chosen so that $e^{-C g}$ is $q$ convex in a neighborhood of $K$. This is possible [cf. 27, II (1.6)].

Since $\phi(x)=1-e^{-c x}$ is a homeomorphism from $[0,1]$ to $\left[0,1-e^{-c}\right]$, (0.4.3) is the same as:

(0.4.5) an open set $B$ of $X$ with smooth boundary can be found such that $E(F, \phi(c)) \subseteq B \subseteq E(F, \phi(d))$, and such that $\pi_{i}(B, A, x)=0$ for any $x$ $\in A$ and $i \leq \operatorname{dim}_{C} X-q$.

Use (0.2.1) and (0.4.4) to choose a $C^{\infty}$ function $\tilde{F}$ on $X$ such that:

a) $\sup _{X}|\tilde{F}-F|<\varepsilon=\min \left\{R_{1} / 4, R_{2} / 4\right\}$ where $R_{1}=\phi(\alpha)-\phi\left(\alpha^{\prime}\right)$ and $R_{2}=\phi(d)-\phi(c)$

b) $\tilde{F}$ is a Morse function in a neighborhood of $K$, and,

c) the Levi form of $\tilde{F}$ has at most $q-1$ non-negative eigenvalues at each point of a neighborhood of $K$.

By (0.2.2) I have:

$$
\begin{aligned}
E(F, \phi(c)) \subseteq E\left(\tilde{F}, \phi(c)+\varepsilon^{\prime}\right) \subseteq E(F, \phi(d)) \\
\text { for } \varepsilon^{\prime} \text { with } \varepsilon \leq \varepsilon^{\prime} \leq \phi(d)-\phi(c)-\varepsilon=R_{2}-\varepsilon
\end{aligned}
$$

and

$$
\begin{aligned}
& E\left(F, \phi\left(\alpha^{\prime}\right)\right) \subseteq E\left(\tilde{F}, \phi\left(\alpha^{\prime}\right)+\varepsilon^{\prime \prime}\right) \subseteq E(F, \phi(\alpha)) \\
& \quad \text { for } \varepsilon^{\prime \prime} \text { with } \varepsilon \leq \varepsilon^{\prime \prime} \leq \phi(\alpha)-\phi\left(\alpha^{\prime}\right)-\varepsilon=R_{1}-\varepsilon .
\end{aligned}
$$

Since $\tilde{F}$ is a Morse function on $K, d \tilde{F}$ equals 0 on only a discrete set of $K$. Thus using (0.4.6) and the fact that $K \supseteq E(F, \phi(c), \phi(d))$, I can 
choose an $\varepsilon^{\prime} \in\left(\varepsilon, R_{2}-\varepsilon\right)$ such that $B=E\left(\tilde{F}, \phi(c)+\varepsilon^{\prime}\right)$ has a smooth boundary and:

$$
E(F, \phi(c)) \subseteq B \subseteq E(F, \phi(d))
$$

Note that the real index of $\tilde{F}$ at any point of $K$ is at least as large as the index of the Levi form of $\tilde{F}$ at the same point. Thus since $E\left(F, \phi\left(\alpha^{\prime}\right)\right.$, $\phi(\alpha)) \subseteq K$, I conclude that $B$ is obtained from $E\left(\tilde{F}, \phi\left(\alpha^{\prime}\right)+\varepsilon^{\prime \prime}\right)$ for $\varepsilon^{\prime \prime} \in$ $\left(\varepsilon, R_{1}-\varepsilon\right)$ by attaching at least $\operatorname{dim}_{C} X-q+1$ cells. Thus:

$$
\begin{aligned}
& \pi_{i}\left(B, E\left(\tilde{F}, \phi\left(\alpha^{\prime}\right)+\varepsilon^{\prime \prime}\right), x\right)=0 \\
& \quad \text { for } i \leq \operatorname{dim}_{C} X-q, x \in A, \text { and } \varepsilon^{\prime \prime} \in\left(\varepsilon, R_{1}-\varepsilon\right) .
\end{aligned}
$$

I have:

$$
A \subseteq E\left(\tilde{F}, \phi\left(\alpha^{\prime}\right)+\varepsilon^{\prime \prime}\right) \subseteq E(F, \phi(\alpha))=E(g, \alpha) \subseteq W \subseteq B
$$

where $W$ was defined right before (0.4.4).

Therefore:

$$
\pi_{i}(A, x) \longrightarrow \pi_{i}\left(E\left(\tilde{F}, \phi\left(\alpha^{\prime}\right)+\varepsilon^{\prime \prime}\right), x\right) \longrightarrow \pi_{i}(W, x) \longrightarrow \pi_{i}(B, x)
$$

for $x \in A$. Using (0.4.9) and the fact that the composition of the first two maps is a bijection for all $j$, I get (0.4.5).

(0.4.10) Corollary. Let $X, A, f,\left\{c_{j}\right\}$ and $\left\{B_{j}\right\}$ be as in (0.4). For all sufficiently large $c_{j}$,

$$
\pi_{i}(A, x) \longrightarrow \pi_{i}\left(B_{j}, x\right)
$$

is an injection for all $i$ and all $x \in A$.

Proof. Looking at the last paragraph of the proof of (0.4), we see that for $c_{j}$ sufficiently large, $B_{j} \subseteq W$ and that:

$$
\pi_{i}(A, x) \longrightarrow \pi_{i}\left(B_{j}, x\right) \longrightarrow \pi_{i}(W, x)
$$

with the composition an isomorphism for all $i$ and all $x \in A$.

(0.5) In $\S 2$ it will be very convenient to work with simplicial complexes and polyhedra. I refer to [30, Chap. 3] as my basic reference. Here I will simply recall the major definitions and facts, assuming the reader knows what a simplicial complex is.

A polyhedron [30, p. 113], $X$, is a space which can be triangulated, i.e. it is the realization of a simplicial complex, $K$. When we want to emphasize $K$ we write $X=|K|$. A polyhedral pair [30, p. 114] $(X, A)$, is 
a pair consisting of a polyhedron $X=|K|$ and a subspace $A=|L|$ where $L$ is a subcomplex of $K$. Letting $(X, A)=(|K|,|L|)$ and $(Y, B)=(|M|,|N|)$ be two polyhedral pairs, a continuous map $f:(X, A) \rightarrow(Y, B)$ is by abuse of language said to be simplicial if it is the realization, $|\varphi|$, of a simplicial map [30, p. 109], $\varphi$ from $(K, L)$ to $(M, N)$.

The following summarizes some of the basic facts I will use repeatedly.

(0.5.1) Proposition. Let $(X, A)=(|K|,|L|)$ and $(Y, B)=(|M|,|N|)$ be two polyhedral pairs and let $Z=|P|$ be a polyhedron.

(0.5.1.1) If $f:(X, A) \rightarrow(Y, B)$ is a continuous map, there exists a simplicial map $g:(X, A) \rightarrow(Y, B)$ associated to a subdivision of $K$ which is homotopic to $f$. If $\left.f\right|_{A}$ is a simplicial map then $g$ can be chosen so that $\left.g\right|_{A}=\left.f\right|_{A}$.

(0.5.1.2.) $A$ is a strong deformation retract of some neighborhood of $A$ in $X$.

(0.5.1.3) Let $f: X \rightarrow Y$ and $h: Z \rightarrow Y$ be simplicial maps, i.e. $f=|\varphi|$ and $h=|\psi|$, and consider the commutative diagram:

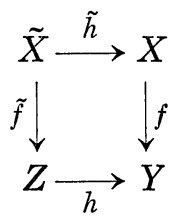

where $\tilde{X}$ is the fibre product of $f$ and $h$, and, $\tilde{f}$ and $\tilde{h}$ are the induced maps. Then $X$ is a polyhedron and $\tilde{f}$ and $\tilde{h}$ are simplicial maps.

Proof. (0.5.1.1) follows from Theorem 8 and the discussion after it in [30, p. 128] and Lemmas 1 and 2 of [30, p. 126].

Next, (0.5.1.2) is Corollary 11 of [30, p. 124].

The last is a straightforward check. Essentially $\tilde{X}$ is the realization of the abstract simplicial complex obtained by taking the fibre product of $\varphi: K \rightarrow M$ and $\psi: P \rightarrow M$ in the category of abstract simplicial complexes.

The next trivial lemma will also be useful.

(0.5.2) Lemma. Let $B$ be the closed unit ball in $\boldsymbol{R}^{n}$. Let $\partial B$ denote its boundary and let $y \in \partial B$. Let $\varphi_{i}: B \rightarrow Y$ with $i \in\{1,2\}$ be two continuous maps into a topological space $Y$. Assume that there exists a continuous map $h: B \rightarrow B$ such that $\left.h\right|_{\partial B}$ is the identity and such that: 


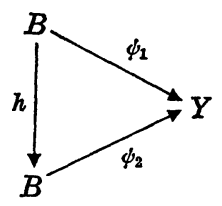

commutes. Then $\phi_{1}$ and $\phi_{2}$ give rise to the same element of $\pi_{n}(Y, Z, x)$ where $\phi_{1}(\partial B) \subseteq \bar{Z}=Z \subseteq Y$ and $x=\phi_{i}(t)$ for some $t \in \partial B$. If $\phi_{1}(\partial B)=\phi_{2}(\partial B)=x$ $\in Y$, then $\phi_{1}$ and $\phi_{2}$ induce the same element of $\pi_{n}(Y, x)$.

Proof. This is an immediate consequence of the fact that:

$$
\text { th }+(1-t) \mathrm{id}_{B} \quad 0 \leq t \leq 1
$$

gives an homotopy between $h$ and $\mathrm{id}_{B}$, the identity map of $B$.

The following form of the Hurewicz-Whitehead theorem is needed.

(0.6) Lemma. Let $f: X \rightarrow Y$ be. a continuous map between connected and simply connected $C W$ complexes. Then

$$
f_{*}: \pi_{j}(X, a) \longrightarrow \pi_{j}(Y, f(a))
$$

is for each $a \in X$, an isomorphism for $j<k$ and an epimorphism for $j=k$ if and only if for each Abelian group $\mathscr{G}$,

$$
f^{*}: H^{j}(Y, \mathscr{G}) \longrightarrow H^{j}(X, \mathscr{G})
$$

is an isomorphism for $j<k$ and an injection for $j=k$.

Proof. By the Hurewicz theorem the homotopy condition is equivalent to:

$$
f_{*}: H_{j}(X, Z) \longrightarrow H_{j}(Y, Z)
$$

is an isomorphism for $j<k$ and a surjection for $j=k$. By considering the mapping cylinder $\mathscr{M}(f)$ of $f: X \rightarrow Y$, one sees by the relative homology sequence that the above is equivalent to:

$$
H_{j}(\mathscr{M}(f), X, Z)=0 \quad \text { for } j \leq k .
$$

The latter is equivalent to:

$$
H_{j}(\mathscr{C}(f), Z)=0 \quad \text { for } 0<j \leq k \text { and } H_{0}(\mathscr{C}(f), Z)=Z
$$

where $\mathscr{C}(f)=\mathscr{M}(f) / X$ is the mapping cone of $f$. The above, by the universal coefficient theorem, implies:

$$
H^{j}(\mathscr{C}(f), \mathscr{G})=0 \quad \text { for } 0<j \leq k
$$


and $H^{0}(\mathscr{C}(f), \mathscr{G})=\mathscr{G}$ for, all coefficient groups $\mathscr{G}$.

Conversely (0.6.2) implies (0.6.1). To see this, assume (0.6.2) and assume $H_{\lambda}(\mathscr{C}(f), Z) \neq 0$ for some $\lambda$ with $0<\lambda \leq k$. Let $\mathscr{G}=Z_{\lambda} / B_{\lambda}$ where $Z_{\lambda}$ is the $\lambda^{\text {th }}$ term of the singular chain complex of $\mathscr{C}(f)$ and $B_{\lambda}$ is the submodule of $Z_{\lambda}$ consisting of boundaries. There is a natural homomorphism of $Z_{\lambda}$ to $\mathscr{G}=Z_{\lambda} / B_{\lambda}$ and it is easy to see that this shows $H^{\lambda}(\mathscr{C}(f), \mathscr{G})$ cannot be zero.

Now note (0.6.2) is equivalent to $H^{j}(\mathscr{M}(f), X, \mathscr{G})=0$ for $j \leq k$ for all Abelian groups, $\mathscr{G}$, which by the relative cohomology sequence is equivalent to:

$$
f^{*}: H^{j}(Y, \mathscr{G}) \longrightarrow H^{j}(X, \mathscr{G})
$$

being an isomorphism for $j<k$ and a surjection for $j=k$ for all Abelian groups $\mathscr{G}$.

\section{§1. Morse theory with parameters}

In this section I prove a parametrized version of (0.4). $X$ always denotes a connected complex manifold and $S$ denotes a connected finite dimensional polyhedron (cf. (0.5)). Let $p: X \times S \rightarrow S$ and $q: X \times S \rightarrow X$ denote the product projections.

$\mathscr{A}$ denotes a closed subset of $X \times S$ and $\mathscr{A}_{s}=p^{-1}(s) \cap \mathscr{A}$. It is assumed that:

(1.0.1) $q\left(\mathscr{A}_{s}\right)$ is an analytic subset of $X$ for all $s \in S$, and $\left.p\right|_{\mathscr{A}}: \mathscr{A} \rightarrow S$ is a proper map.

It is assumed further that there is a neighborhood $U$ of $\mathscr{A}$ in $X \times S$ and a real valued non-negative continuous function $f$ on $U-\mathscr{A}$ such that:

(1.0.2) $\lim _{n \rightarrow \infty} f\left(x_{n}\right)=\infty$ for any sequence $\left\{x_{n}\right\} \subseteq U-\mathscr{A}$ converging to a point of $\mathscr{A}$, and

(1.0.3) for each $t \in S, f$ is asymptotically $\lambda$ convex on $U \cap p^{-1}(t)-\mathscr{A}_{t}$ where $\lambda \leq \operatorname{dim}_{C} X$.

(1.1) Proposition. $\mathscr{A}_{t}$ is non-empty for all $t \in S$ if it is non-empty for one $t$ in $S$.

Proof. This is a consequence of the maximum principle. For a proof see $[28,(1.1)]$ with $A=X$ and $B=\mathscr{A}$. 
(1.2) Proposition. Let $t \in S$. There exists an open set $B$ of $X$ with $a$ smooth boundary and a neighborhood $W$ of $t$ in $S$ such that:

$$
\begin{aligned}
& \mathscr{A} \cap p^{-1}(W) \subseteq B \times W, \\
& \pi_{i}\left(B \times\{p(x)\}, \mathscr{A}_{p(x)}, x\right)=0 \\
& \quad \text { for } i \leq \operatorname{dim}_{C} X-\lambda \text { and each } x \in \mathscr{A} \cap p^{-1}(w),
\end{aligned}
$$

and,

$$
\begin{aligned}
\pi_{i}\left(\mathscr{A}_{p(x)}, x\right) & \longrightarrow \pi_{i}(B \times\{p(x)\}, x) \\
& \text { is an injection for all } i \text { and all } x \in \mathscr{A}_{t} .
\end{aligned}
$$

Proof. Given $t \in S$, I claim that there is a relatively compact open set $V$ of $X$ and a relatively compact neighborhood $K$ of $t$ such that:

$$
V \times\{s\} \supseteq \mathscr{A}_{s} \quad \text { for all } s \in K \text { and } \bar{V} \times \bar{K} \subseteq U \text {, }
$$

and

(1.2.5) there exists a $c \in \boldsymbol{R}$ such that $E(s, c)=\{(x, s) \in V \times\{s\}-$ $\left.\mathscr{A}_{s} \mid f(x, s)>c\right\} \cup \mathscr{A}_{s}$ is relatively compact in $V \times\{s\}$.

These are easy; for a proof see [28, (1.2.1) and (1.2.2)].

Let $g(x, s)$ denote the continuous extension of $e^{-f}$ to $V \times K$ so that $g^{-1}(0)=\mathscr{A} \cap p^{-1}(K)$.

Now consider $g(y, s)$ as a function $g_{s}(y)$ on $V$. After shrinking $K$ one has (using the notation of (0.2.2)):

(1.2.6) $E\left(g_{s}, d\right)$ is relatively compact in $V$ for all $s \in K$ and $d \leq e^{-c}$ where $d \in R$ and $c$ is as in (1.2.5), and,

(1.2.7) there exists an $\varepsilon>0$ and a $d^{\prime}<d-\varepsilon$ such that:

$$
\begin{aligned}
q\left(\mathscr{A}_{s}\right) & \subseteq E\left(g_{t}, d^{\prime}-4 \varepsilon\right) \subseteq E\left(g_{t}, d^{\prime}-3 \varepsilon\right) \subseteq E\left(g_{s}, d^{\prime}-2 \varepsilon\right) \\
& \subseteq E\left(g_{s}, d^{\prime}-\varepsilon\right) \subseteq E\left(g_{t}, d^{\prime}\right) \subseteq E\left(g_{t}, d^{\prime}+\varepsilon\right) \quad \text { for all } s \in K .
\end{aligned}
$$

The above are easy consequences of $(0.2 .2)$; for more details see [28, (1.2.5) and (1.2.6)].

By (0.4) and (0.4.10) there exist open sets $B_{1}(s), B_{1}(t)$, and $B_{2}(t)$ with smooth boundaries such that:

$$
\begin{aligned}
& E\left(g_{t}, d^{\prime}-4 \varepsilon\right) \subseteq B_{1}(t) \subseteq E\left(g_{t}, d^{\prime}-3 \varepsilon\right), \\
& E\left(g_{t}, d^{\prime}\right) \subseteq B_{2}(t) \subseteq E\left(g_{t}, d^{\prime}+\varepsilon\right), \text { and } \\
& E\left(g_{s}, d^{\prime}-2 \varepsilon\right) \subseteq B_{1}(s) \subseteq E\left(g_{s}, d^{\prime}-\varepsilon\right),
\end{aligned}
$$




$$
\begin{aligned}
& \pi_{j}\left(B_{i}(t), q\left(\mathscr{A}_{t}\right), x\right)=0=\pi_{j}\left(B_{1}(s), q\left(\mathscr{A}_{s}\right), y\right) \\
& \quad \text { for } j \leq \operatorname{dim}_{C} X-\lambda, x \in q\left(\mathscr{A}_{t}\right), y \in q\left(\mathscr{A}_{s}\right) \text {, and } i=1 \text { or } 2,
\end{aligned}
$$

and

$$
\begin{array}{r}
\pi_{j}\left(q\left(\mathscr{A}_{t}\right), x\right) \longrightarrow \pi_{j}\left(B_{i}(t), x\right) \text { is an injection for } i=1 \text { or } 2, \\
\text { for all } j, \text { and } x \in q\left(\mathscr{A}_{t}\right) .
\end{array}
$$

Combine (1.2.7) and (1.2.8) into a sequence of inclusions. Using (1.2.9) and (1.2.10) on this sequence, the result immediately follows with $B=$ $B_{2}(t)$.

(1.3) Corollary. Assume the assumptions of (1.2) and also assume that $\mathscr{A}$ is a polyhedron and $f=\left.p\right|_{\mathscr{A}}: \mathscr{A} \rightarrow S$ is a simplicial map. If $\operatorname{dim}_{C} X$ $>\lambda$ then $f$ factors as $g \circ h$ where $h: \mathscr{A} \rightarrow Z$ is a simplicial map with connected fibres between polyhedra and $g: Z \rightarrow S$ is a finite covering map. Further given any $x \in \mathscr{A}$, there is a neighborhood $V$ of $h(x)$ in $Z$ and an open set $U \supseteq h^{-1}(V)$ such that:

$$
\pi_{j}\left(U, h^{-1}(h(v)), v\right)=0 \quad \text { for } j \leq \operatorname{dim}_{C} X-\lambda \text { and all } v \in h^{-1}(V) .
$$

Proof. To see that the factorization $g \circ h$ exists with $g$ a finite covering it suffices to work locally on $S$. Let $W$ be a neighborhood of a point $f(x)=t \in S$ which is contractible and small enough so that (1.2.1), (1.2.2), and (1.2.3) hold.

Let $F$ be a set with one point corresponding to each connected component of $B$. The factorization $\left.p\right|_{B}: B \times W \rightarrow W$ into $a: B \times W \rightarrow F \times W$ and $b: F \times W \rightarrow W$ induces a factorization $b \circ \tilde{h}$ of $\left.p\right|_{\left[\propto p^{-1(W)]}\right.}$ where $\tilde{h}=$ $\left.a\right|_{\left[\mathscr{A}_{\cap} p^{-1}(W)\right]}$. Since $\mathscr{A}_{t}$ is a compact analytic space it has finitely many components. By (1.2.2) and $\operatorname{dim}_{C} X>\lambda$ each component of $B$ contains precisely one connected component of $q\left(\mathscr{A}_{s}\right)$ for any $s \in W$. By the last two sentences $F$ is finite and $\tilde{h}$ is surjective with connected fibres.

Thus we have a global factorization $f=g \circ h$ where $h: \mathscr{A} \rightarrow Z$ is a continuous map with connected fibres onto a topological space $Z$ and where $g: Z \rightarrow S$ is a covering projection. Since $g$ is a covering and $f$ is simplicial, the simplicial structure of $S$ induces the structure of a polyhedron on $Z$ in such a way that $g$ and $h$ are simplicial.

Now let us turn to (1.3.1).

Letting $W$ be as above, it is clear that we can assume without loss of generality that $f$ has connected fibres. Let $U$ be a neighborhood of $f^{-1}(t)$ that is a strong deformation retract of $f^{-1}(t)$. Let $V$ be a neighbor- 
hood of $t$ such that $f^{-1}(V) \subseteq U$. This is possible by (0.5.1.2) and the fact that $f$ is proper. Now consider the diagram with $q_{v}=\left.q\right|_{U}$ and $q_{v}=$ $\left.q\right|_{f-1(f(v))}$ :

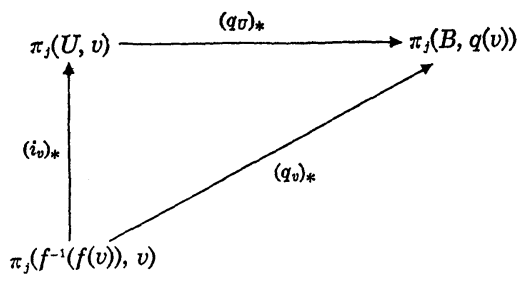

Letting $v=t$ we see by (1.2.2), (1.2.3) and the definition of $U$ that $\left(q_{U}\right)_{*}$ is an isomorphism for $j \leq k=\operatorname{dim}_{C} X-\lambda$, since $\left(q_{t}\right)_{*}$ and the inclusion $\left(i_{t}\right)_{*}$ are. Since $U$ is connected, $\left(q_{U}\right)_{*}$ has this property for all $v \in U$.

By (1.2.2) applied to $\left(q_{v}\right)_{*}$ and the above, we conclude that

$$
\pi_{j}\left(U, f^{-1}(f(v)), v\right)=0 \quad \text { for } j \leq k \text { and } v \in f^{-1}(V) .
$$

\section{§2. A long exact homotopy sequence}

(2.1) Definition. Let $f$ be a simplicial map from a connected polyhedron $X$ onto a polyhedron $Y$. We say $f$ is a $k$ quasi-fibration if:

(2.1.1) there is a polyhedron $Z$ such that $f$ factors as $q \cdot p$ where $p$ : $X \rightarrow Z$ is a proper simplicial surjection with connected fibres and $q: Z \rightarrow$ $Y$ is a covering map.

(2.1.2) given any $z \in Z$, there exist a contractible open set $V$ of $Z$ containing $z$ and an open set $U$ of $X$ containing $p^{-1}(V)$ such that $\pi_{j}\left(U, p^{-1}(p(x)), x\right)=0$ for $j \leq k$ and $x \in p^{-1}(V)$.

(2.2) Remark. The above codifies (1.3) in a form useful for applications. It is reminiscent of the Dold-Thom [10] definition of quasifaserungen. Unfortunately it is a priori weaker; this section would be a corollary of [10] if we knew that the $V$ of the preceding definition could be chosen so that $\pi_{j}\left(p^{-1}(V), p^{-1}(p(x)), x\right)=0$ for $j \leq k$ and all $x \in p^{-1}(V)$.

The next result is the object of this section.

(2.3) Proposition. Let $f: X \rightarrow Y$ be a $k$ quasi-fibration. Given any point $x \in X$ one has that:

$$
\pi_{j}(Y, f(x)) \longleftarrow \pi_{j}\left(X, f^{-1}(f(x)), x\right)
$$


is an isomorphism for $j \leq k$ and a surjection for $j=k+1$. In particular, one has the long exact homotopy sequence

$$
\begin{aligned}
\pi_{k}\left(f^{-1}(f(x)), x\right) \longrightarrow \pi_{k}(X, x) \longrightarrow \pi_{k}(Y, f(x)) \longrightarrow \\
\cdots \longrightarrow \pi_{1}(Y, f(x)) \longrightarrow \pi_{0}\left(f^{-1}(f(x)), x\right) \longrightarrow 0 .
\end{aligned}
$$

The sequence (2.3.2) is functorial with respect to morphisms of $k$ quasifibrations.

Proof. If (2.3.1) is proven, then the rest of the proof will follow from the long exact homotopy sequence of a pair.

The idea is that [30, p. 100] any continuous map is homotopic to a map of Serre fibrations. This gives us a commutative diagram

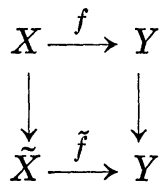

where the vertical arrows are homotopy equivalences and $\tilde{f}$ is a Serre fibration. Since there is a long exact homotopy sequence for $\tilde{f}$, we would be done if the map from a fibre of $f$ to a fibre of $\tilde{f}$ induced an isomorphism of homotopy groups in the proper range. It is easy to use the Leray spectral sequence, (2.1.2), and the Hurewicz-Whitehead theorem (0.6) to prove this if everything is simply connected. But we cannot assume this; so we prove a sequence of lemmas leading up to (2.3.8), where we prove (2.3) for contractible $Y$ by using a modified form of the above idea. Then we use this partial result to get the full result in quick order. I suggest that the reader look over the statements of the various lemmas to get a more precise idea of the proof before he works through it in detail.

(2.3.3) Lemma. Let $\gamma:[0,1] \rightarrow Y$ be a simplicial map with $\gamma(0)=f(x)$. Let $f^{\prime}: X^{\prime} \rightarrow[0,1]$ be the pullback of $f$ by $\gamma$. Let $x^{\prime} \in X^{\prime}$ be the point in $f^{\prime-1}(0)$ corresponding to $x$ in $X$. There exists a simplicial map $\gamma^{\prime}:[0,1] \rightarrow$ $X^{\prime}$ with $\gamma^{\prime}(0)=x^{\prime}$ and $f^{\prime}\left(\gamma^{\prime}(1)\right)=1$. The map $f_{*}: \pi_{1}\left(X, f^{-1}(f(x)), x\right) \rightarrow \pi_{1}(Y, f(x))$ is a surjection.

Proof. In showing that $\gamma^{\prime}$ exists, it can be assumed that $f$ and hence $f^{\prime}$ have connected fibres. Indeed, replace $\gamma$ by the unique lift $\tilde{\gamma}:[0,1] \rightarrow Z$ of $\gamma$, with $\tilde{\gamma}(0)=p(x)$.

Now $X^{\prime}$ is a compact polyhedron by (0.5.1.3). If it is connected, it 
will be path connected and $\gamma^{\prime}$ will then clearly exist. To see that it is connected, assume otherwise. Thus let $X^{\prime}=U \cup V$ where $U \cap V=\phi$ and $U$ and $V$ are non-trivial open sets. One quickly concludes that $U=$ $f^{\prime-1}(A)$ and $V=f^{\prime-1}(B)$ for some disjoint sets $A$ and $B$ of $[0,1]$. Since $U$ and $V$ are closed and compact, so are $A$ and $B$. This contradiction implies $X^{\prime}$ is connected.

Now let $\gamma$ represent an element of $\pi_{1}(Y, f(x))$. The map $\gamma^{\prime \prime}:[0,1] \rightarrow X$ that $\gamma^{\prime}$ induces represents an element of $\pi_{1}\left(X, p^{-1}(p(x)), x\right)$. Considering (0.5.2) and the commutative diagram:

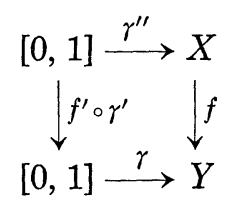

we see that the lemma is proven.

By (2.3.3) $k$ can be and is assumed $\geq 1$ without loss of generality.

(2.3.4) LEMMA. In proving (2.3), it can be assumed without loss of generality that $f$ has connected fibres.

Proof. Let $f=q \circ p$ as in (2.1) and consider the commutative diagram:

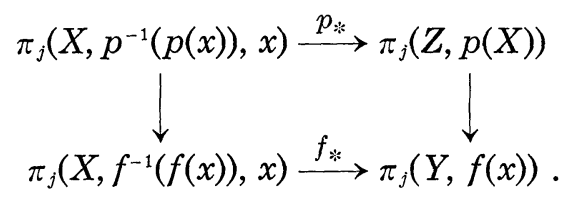

The vertical arrows are of course isomorphisms for $j>1$ and thus, for $j>1, p_{*}$ is an isomorphism if and only if $f_{*}$ is. Let $j=1$ and assume that $p_{*}$ is an isomorphism. By (2.3.3), $f_{*}$ is a surjection. Assume it is not an injection. Let $\gamma:[0,1] \rightarrow X$ with $\gamma(0)=x$ be a simplicial map representing an element of $\pi_{1}\left(X, f^{-1}(f(x)), x\right)$ going to zero under $f_{*}$. Since $f \circ \gamma$ represents the trivial loop in $Y$, one concludes there is a unique lift $\gamma^{\prime}:[0,1] \rightarrow Z$ of $f \circ \gamma$ satisfying $\gamma^{\prime}(1)=\gamma^{\prime}(0)=p(x)$ and representing the trivial loop in $Z$. Noting that $\gamma^{\prime}=p \circ \gamma$ we see that $\gamma$ represents an element of $\pi_{1}\left(X, p^{-1}(p(x)), x\right)$. Since $p_{*}(\gamma)$ is trivial and $p_{*}$ is an isomorphism, it follows that $\gamma$ is trivial as an element of $\pi_{1}\left(X, p^{-1}(p(x)), x\right)$ and hence of $\pi_{1}\left(X, f^{-1}(f(x)), x\right)$.

(2.3.5) Lemma. If $k \geq 1$ in (2.3), then $\pi_{1}(Y, f(x)) \approx \pi_{1}\left(X, f^{-1}(f(x)), x\right)$ if $Y$ is simply connected. 
Proof. We can by (2.3) assume without loss of generality that $f$ has connected fibres. Using the exact homotopy sequence of the pair $\left(X, f^{-1}(f(x))\right)$, and the facts that $Y$ is simply connected, and $f^{-1}(f(x))$ is connected, we see that we must show:

$$
\pi_{1}\left(f^{-1}(f(x)), x\right) \longrightarrow \pi_{1}(X, x) \longrightarrow 0 .
$$

I proceed to do this with a trick I learned from [5]. Let $g: \tilde{X} \rightarrow X$ be the connected covering associated to the image of $\pi_{1}\left(f^{-1}(f(x)), x\right)$ in $\pi_{1}(X, x)$. Let $\mathscr{S}$ be the sheaf of germs of sections of $g$. Let $f_{*}(\mathscr{S})$ be the direct image sheaf of $\mathscr{S}$ under $f$. Recall that $\left.f_{*}(\mathscr{S})\right|_{y}=\lim _{G \in \mathscr{U}(y)} \Gamma\left(f^{-1}(G), \mathscr{S}\right)$ where $\mathscr{U}(y)$ denotes the set of open sets of $Y$ containing $y$. Now $f_{*}(\mathscr{S})$ is locally constant. To see this, let $U$ and $V$ with $U \supseteq f^{-1}(V) \supseteq f^{-1}(y)$ be the open sets of (2.1.2) for a point $y \in Y$. Now (2.1.2) implies that the images of $\pi_{1}(U, z), \pi_{1}\left(f^{-1}(V), z\right)$, and $\pi_{1}\left(f^{-1}(f(z)), z\right)$ in $\pi_{1}(X, z)$ are the same for $z \epsilon$ $f^{-1}(V)$. Thus any section of $g: \tilde{X} \rightarrow X$ over $f^{-1}(f(z))$ extends to a section of $g: \tilde{X} \rightarrow X$ over $f^{-1}(V)$. Thus $\left.\Gamma\left(f^{-1}(V), \mathscr{S}\right) \approx \Gamma\left(V, f_{*}(\mathscr{S})\right) \approx f_{*}(\mathscr{S})\right|_{f(z)}$ for all $z \in f^{-1}(V)$. Thus $f_{*}(\mathscr{S})$ is locally constant and hence constant, since $Y$ is simply connected. Now if (2.3.5.1) is false, then $\left.f_{*}(\mathscr{S})\right|_{f(x)}$ has at least two elements and therefore $\Gamma\left(Y, f_{*}(\mathscr{S})\right) \approx \Gamma^{*}(X, \mathscr{S})$ has a non-trivial element. This contradicts the fact that $g: \tilde{X} \rightarrow X$ is a non-trivial connected cover.

(2.3.6) Lemma. Let $f: X \rightarrow Y$ be a $k$ quasi fibration with $k>1$ and connected fibres. Given any point $y \in Y$, there is a neighborhood $V$ of $y$ such that given any contractible open set $W$ of $V$, we have:

$$
\pi_{1}\left(f^{-1}(W), z\right) \approx \pi_{1}\left(f^{-1}(f(z)), z\right)
$$

for any $z \in f^{-1}(W)$.

Proof. Let $V$ and $U$ be as in (2.1.2). Let $W$ be any contractible open set of $V$. By (2.1.2), the map $\pi_{1}\left(f^{-1}(f(z)), z\right) \rightarrow \pi_{1}(U, z)$ is an injection for $z \in f^{-1}(V)$. Thus the map $\pi_{1}\left(f^{-1}(f(z)), z\right) \rightarrow \pi_{1}\left(f^{-1}(W), z\right)$ is an injection for $z \in f^{-1}(W)$. Now by (2.3.5) the map $\pi_{1}\left(f^{-1}(f(z)), z\right) \rightarrow \pi_{1}\left(f^{-1}(W), z\right)$ is a surjection for $z \in f^{-1}(W)$.

(2.3.7) Lemma. Let $B$ be the closed unit ball of $\boldsymbol{R}^{n}$. Given an $\varepsilon>0$, there exists a set $\left\{F_{1}, \cdots, F_{N}\right\}$ of open sets of $B$ for some $N>0$ such that: $E_{i}=\bigcup_{j \leq i} F_{j}$ is contractible for each $i$,

(2.3.7.2) the diameter of each $F_{i}$ in the Euclidean metric on $B$ is $<\varepsilon$, 


$$
\begin{aligned}
& B=E_{N}=\bigcup_{j \leq N} F_{j} \text { and for all } i, E_{i} \cap F_{i+1} \text { is contractible and } \\
& \text { non-empty. }
\end{aligned}
$$

Proof. I will assume $n=3$ for simplicity of notation. Note that $B$ is homeomorphic to $I \times I \times I$ where $I$ is the unit interval. Let $a, b$, and $c$ be positive integers less than or equal to a positive integer $n$. Let $F_{n}(a, b, c)$ denote the set

$$
\begin{aligned}
& \left\{(x, y, z) \in I \times I \times I \mid \frac{a-1}{n}-\frac{1}{3 n}<x<\frac{a}{n}+\frac{1}{3 n},\right. \\
& \left.\quad \frac{b-1}{n}-\frac{1}{3 n}<y<\frac{b}{n}+\frac{1}{3 n}, \frac{c-1}{n}-\frac{1}{3 n}<z<\frac{c}{n}+\frac{1}{3 n}\right\} .
\end{aligned}
$$

Now choose $n$ large enough so that the $F_{n}(a, b, c)$ satisfy (2.3.7.2). Order the set of triples $\{(a, b, c) \mid a, b, c$ are positive integers less than or equal to a positive integer $n\}$ by setting $\left(a_{1}, b_{1}, c_{1}\right) \leq\left(a_{2}, b_{2}, c_{2}\right)$ if $a_{1} \leq a_{2}$, or if $a_{1}$ $=a_{2}$ and $b_{1} \leq b_{2}$, or if $a_{1}=a_{2}$ and $b_{1}=b_{2}$ and $c_{1} \leq c_{2}$. Using this ordering, let $F_{j}$ denote the $j$ th element of the set of $F_{n}(a, b, c)$ with $a, b$ and $c$ less than or equal to $n$. Clearly the $F_{j}$ satisfy (2.3.7.1)-(2.3.7.3).

(2.3.8) Lemma. Assume $Y$ is homeomorphic to the unit ball, $B$, in $\boldsymbol{R}^{M}$ for some $M \geq 1$ and that $f$ has connected fibres. Then (2.3.1) holds.

Proof. By (2.3.5) it can be assumed that $k>1$. First let me show that:

$$
\pi_{1}\left(f^{-1}(f(x)), x\right) \longrightarrow \pi_{1}(X, x)
$$

is an isomorphism for all $x \in X$.

Surjection was shown in (2.3.5).

Use Lemma (2.3.6) and (2.3.7) and the compactness of $B$ to choose contractible open sets $\left\{F_{1}, \cdots, F_{N}\right\}$ of $B$ such that:

$$
E_{i}=\bigcup_{j \leq i} F_{j} \text { is contractible for each } i,
$$

(2.3.8.3) $B=E_{N}$ and $E_{i} \cap F_{i+1}$ is contractible and non-empty,

and

$$
\pi_{1}\left(f^{-1}(f(z)), z\right) \longrightarrow \pi_{1}\left(f^{-1}(\mathscr{W}), z\right)
$$

is an isomorphism for each contractible open set $\mathscr{W} \subseteq F_{\text {f }}$ where $j \leq N$ and each $z \in f^{-1}(\mathscr{W})$.

Now assume as an induction hypothesis that given an $N^{\prime}<N$ we have 


$$
\pi_{1}\left(f^{-1}(f(z)), z\right) \longrightarrow \pi_{1}\left(f^{-1}\left(E_{N^{\prime}}\right), z\right)
$$

is an isomorphism for all $z \in f^{-1}\left(E_{N^{\prime}}\right)$.

Let $z \in f^{-1}\left(E_{N^{\prime}} \cap F_{N^{\prime}+1}\right)$. By (2.3.8.4) applied to $\mathscr{W}=F_{N^{\prime}+1}$ and $E_{N^{\prime}} \cap$ $F_{N^{\prime}+1}$, by (2.3.8.5) and by the Seifert-van Kampen theorem, one concludes that:

$$
\pi_{1}\left(f^{-1}(f(z)), z\right) \longrightarrow \pi_{1}\left(f^{-1}\left(E_{N^{\prime}+1}\right), z\right)
$$

is an isomorphism for $z \in f^{-1}\left(F_{N^{\prime}+1} \cap E_{N^{\prime}}\right)$. Now as soon as we show that (2.3.8.6) holds for all $z \in f^{-1}\left(E_{N^{\prime}+1}\right)$ then by induction we will have shown $\pi_{1}\left(f^{-1}(f(x)), x\right)$ injects into $\pi_{1}(X, x)$ for all $x \in X$ and will have established (2.3.8.1). Thus we need only the claim:

(2.3.8.7) ClaIm. Let $f: X \rightarrow Y$ be a $k$ quasi-fibration with connected fibres. If $k>j \geq 1$, then $\pi_{j}\left(f^{-1}(f(x)), x\right)$ injects into $\pi_{j}(X, x)$ for one $x \in X$ if and only if it injects for each $x \in X$.

Proof of Claim. Indeed using (2.1.2) we note that the condition of being an injection holds for $x \in f^{-1}(y)$ where $y$ belongs to open set $W$ of points of $Y$. Considering (2.1.2) at a point $\omega \in \bar{W}-W$ where it is not an injection, we get an immediate contradiction.

Now let $g: \tilde{X} \rightarrow X$ denote the simply connected cover of $X$. Let $\tilde{f}: \tilde{X}$ $\rightarrow B$ denote the map $f \circ g$. By (2.3.8.1) it has connected and simply connected fibres. Let $U$ and $V$ be as in (2.1.2) for some $y \in B$. By taking the inverse image of $U$ under $g$ we get an open set $\tilde{U}$ such that:

$$
\tilde{U} \supseteq \tilde{f}^{-1}(V)
$$

and

(2.3.8.9) given any $z \in \tilde{f}^{-1}(V)$ we have that $\pi_{j}\left(\tilde{U}, \tilde{f}^{-1}(\tilde{f}(z)), z\right)=0$ for $j \leq k$.

Now we see that given any Abelian group $\mathscr{G}$, we have by (0.6) that for $z \in \tilde{f}^{-1}(V)$ :

$$
H^{j}\left(\tilde{U}, \tilde{f}^{-1}(\tilde{f}(z)), \mathscr{G}\right)=0 \quad \text { for } j \leq k .
$$

Now let me show that

$$
\left.\tilde{f}_{(j)}(\mathscr{G})\right|_{y}=H^{j}\left(\tilde{f}^{-1}(y), \mathscr{G}\right)
$$

for any $y \in B$ where $\tilde{f}_{(j)}(\mathscr{G})$ denotes the $j$ th direct image sheaf of $\mathscr{S}$. This follows since we could use (0.5.1.2) and the properness of $f$ to choose a cofinal set of open sets, $\left\{U_{\lambda}\right\}$ around $f^{-1}(y)$ such that $f^{-1}(y)$ was a strong 
deformation retract of each $U_{\lambda}$. Now $\tilde{f}^{-1}(y)$ is a strong deformation retract of each $g^{-1}\left(U_{\lambda}\right)$ and given any open set $\mathscr{H} \ni y$, there is a $U_{\lambda}$ with $\tilde{f}^{-1}(y) \subseteq$ $g^{-1}\left(U_{\lambda}\right) \subseteq \tilde{f}^{-1}(\mathscr{H})$. Thus (2.3.8.11) is shown.

Now note:

(2.3.8.12) $\tilde{f}_{(j)}(\mathscr{G})$ is constant for $j<k$, and

$$
\left.0 \longrightarrow H^{0}\left(B, \tilde{f}_{(k)}(\mathscr{G})\right) \longrightarrow \tilde{f}_{(k)}(\mathscr{G})\right|_{y} \quad \text { for all } y \in B .
$$

The first assertion is an immediate consequence of (2.3.8.10) and (2.3.8.11).

To see the second assertion, note that the first assertion and the contractibility of $B$ imply by the Leray spectral sequence that $H^{0}\left(B, \tilde{f}_{(k)}(\mathscr{G})\right)$ $=H^{k}(\tilde{X}, \mathscr{G})$. Let $e$ be a non-zero element of $H^{0}\left(B, \tilde{f}_{(k)}(\mathscr{G})\right)$. Assume $e$ goes to zero in $\left.\tilde{f}_{(k)}(\mathscr{G})\right|_{y}$ for $y \in B$. Choose a strong deformation retract $g^{-1}\left(U_{\lambda}\right)$ of $\tilde{f}^{-1}(y)$ as in the paragraph after (2.3.8.11). Using this and (2.3.8.11), $\left.H^{k}\left(g^{-1}\left(U_{\lambda}\right), \mathscr{G}\right) \approx H^{k}\left(\tilde{f}^{-1}(y), \mathscr{G}\right) \approx \tilde{f}_{(k)}(\mathscr{G})\right|_{y}$. Thus, noting that

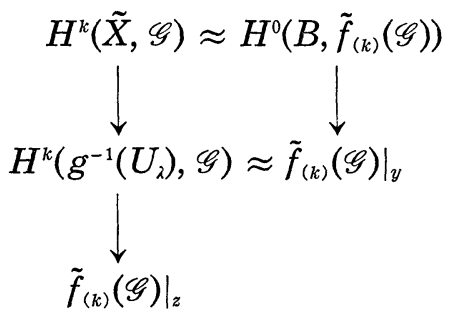

where $f^{-1}(z) \in U_{\lambda}$, we see that $e$ must go to zero in $\left.\tilde{f}_{(k)}(\mathscr{G})\right|_{z}$ for $z$ in a neighborhood of $y$. Thus the set $F$ of $y$ such that $e$ goes to 0 in $\left.\tilde{f}_{(k)}(\mathscr{G})\right|_{y}$ is open in $B$. Using (2.3.8.10), $F$ is seen to be closed also. Thus using the fact that $B$ is connected, $F=B$ or the empty set. If $e$ is non-zero than $F$ must be the empty set.

Now recall [30, p. 100] that there exists a Serre fibration $s: \mathscr{S} \rightarrow B$ where $\mathscr{S}$ is some $C W$ complex and a continuous map $\mu: \tilde{X} \rightarrow \mathscr{S}$ such that

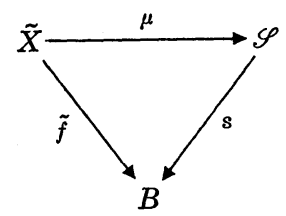

commutes. Further $\mu$ is an homotopy equivalence and thus $\mathscr{S}$ is simply connected, since $\tilde{X}$ is simply connected. From the long exact homotopy sequence of a Serre fibration, the fact that $\mu$ is a homotopy equivalence, and the fact that $B$ is contractible, we conclude that the fibres of $s$ are simply connected. 
Let $E_{2}^{a b}(1)=H^{a}\left(B, s_{(b)}(\mathscr{G})\right)$ and $E_{2}^{a b}(2)=H^{a}\left(B, \tilde{f}_{(b)}(\mathscr{G})\right)$ where $\mathscr{G}$ is an arbitrary Abelian group.

We have, by means of (2.3.8.13), a morphism $\mu^{*}: E_{2}^{a b}(1) \rightarrow E_{2}^{a b}(2)$ between these Leray spectral sequences, which gives rise to an isomorphism on the $E_{\infty}$ terms, since $\mu$ is an homotopy equivalence. Now $\tilde{f}_{(b)}(\mathscr{G})$ is a constant sheaf for $b<k$ by (2.3.8.12) and $s_{(b)}(\mathscr{G})$ is a constant sheaf for all $b$ since $s$ is a Serre fibration. Thus $E_{2}^{a b}(i)=0$ if $a \neq 0$ and $b<k$ for $i=1$ and $i=2$ since $B$ is contractible. Thus $E_{2}^{0 \lambda}(i)=\oplus_{\alpha+\beta=\lambda} E_{\infty}^{\alpha \beta}(i)$ for $\lambda \leq k$. Thus we conclude that:

$$
\mu^{*}:\left.\left.s_{(\lambda)}(\mathscr{G})\right|_{y} \longrightarrow \tilde{f}_{(\lambda)}(\mathscr{G})\right|_{y}
$$

is an isomorphism for $\lambda<k$ and an injection for $\lambda=k$. The latter follows from the fact $(2.3 .8 .12)$ that:

$$
\left.\left.s_{(k)}(\mathscr{G})\right|_{y} \approx H^{0}\left(B, s_{(k)}(\mathscr{G})\right)\right)\left.\approx H^{0}\left(B, \tilde{f}_{(k)}(\mathscr{G})\right) \longrightarrow \tilde{f}_{(k)}(\mathscr{G})\right|_{y}
$$

is an injection. Now this implies:

$$
\mu^{*}: H^{\lambda}\left(s^{-1}(y), \mathscr{G}\right) \longrightarrow H^{\lambda}\left(\tilde{f}^{-1}(y), \mathscr{G}\right)
$$

is for all $y \in B$ an isomorphism for $\lambda<k$ and an injection for $j=k$. Thus since $\mathscr{G}$ is arbitrary and all fibres are connected and simply connected, we conclude from (0.6) that:

$$
\mu_{*}: \pi_{j}\left(\tilde{f^{-1}}(\tilde{f}(x)), x\right) \longrightarrow \pi_{j}\left(s^{-1}(s(\mu(x))), \mu(x)\right)
$$

is for all $x \in \tilde{X}$, an isomorphism for $j<k$ and a surjection for $j=k$.

Now consider the long exact sequences of the pairs $\left(\tilde{X}, \tilde{f}^{-1}(\tilde{f}(x))\right)$ and $\left(\mathscr{S}, s^{-1}(s(\mu(x)))\right)$, which for simplicity I denote $\left(\tilde{X}, F_{1}\right)$ and $\left(\mathscr{S}, F_{2}\right)$ respectively:

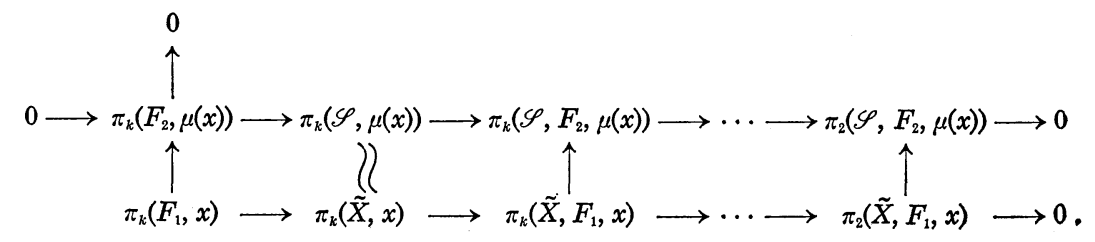

Now using (2.3.8.14), (2.3.8.15) and the five lemma, we conclude that:

$$
\pi_{j}\left(\tilde{X}, F_{1}, x\right) \longrightarrow \pi_{j}\left(\mathscr{S}, F_{2}, \mu(x)\right)
$$

is an isomorphism for all $j \leq k$. And by noting the fact that: 


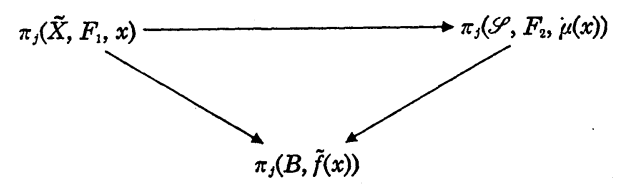

commutes and that the right vertical arrow is an isomorphism, we are finished.

(2.3.9) Lemma. Let $g: W^{\prime} \rightarrow Y$ be a simplicial map from a polyhedron to $Y$. Assume $f$ has connected fibres. Let $f^{\prime}: X^{\prime} \rightarrow W^{\prime}$ be the pullback of $f: X \rightarrow Y$. Then $f^{\prime}: X^{\prime} \rightarrow W^{\prime}$ is a $k$ quasi-fibration.

Proof. By (0.5.1.3) $X^{\prime}$ is in a natural way a polyhedron and $f^{\prime}$ is a simplicial map. Note that $f^{\prime}$ has connected fibres. Let $y \in W^{\prime}$ and let $V$ and $U$ be as in (2.1.2) for $g(y)=z$. Let $\mathscr{W}$ and $\mathscr{V}$ be neighborhoods of $f^{\prime-1}(y)$ in $X^{\prime}$ and $y$ in $W^{\prime}$ respectively such that:

(2.3.9.1) $f^{\prime-1}(y)$ is a strong deformation retract of $\mathscr{W}, \mathscr{V}$ is contractible, and $f^{\prime-1}(\mathscr{V}) \subseteq \mathscr{W}$, and

$$
g\left(f^{\prime}(\mathscr{W})\right) \subseteq V
$$

Note that (2.3.9.1) is possible by (0.5.1.2). Now let $g^{\prime}: X^{\prime} \rightarrow X$ be the map induced by $g$. We have the commutative diagram:

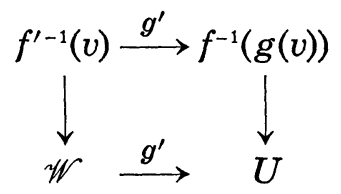

for $v \in \mathscr{V}$. Now the top arrow is a homeomorphism and the left vertical arrow is a homotopy equivalence when $v=y$. Thus, letting $v=y$, we conclude from (2.1.2) that:

$$
g_{*}^{\prime}: \pi_{j}(\mathscr{W}, x) \longrightarrow \pi_{j}\left(U, g^{\prime}(x)\right)
$$

is, for $x \in f^{\prime-1}(y)$, an isomorphism when $j<k$ and a surjection when $j=$ $k$. Since $f$ has connected fibres, it follows that $f^{\prime-1}(y) \approx f^{-1}(g(y))$ is connected and hence $\mathscr{W}$ and $U$ are connected. Thus since $\mathscr{W}$ is a polyhedron it is pathwise connected. It follows that $g_{*}^{\prime}$ is an isomorphism in (2.3.9.4) for $j<k$ and a surjection for $j=k$ for all $w \in \mathscr{W}$. The desired vanishing of $\pi_{j}\left(\mathscr{W}, f^{\prime-1}\left(f^{\prime}(w)\right), w\right)$ for $w \in f^{\prime-1}(\mathscr{V})$ follows from the last sentence and (2.1.2) applied to (2.3.9.3). 
Consider:

$$
\pi_{j}\left(X, f^{-1}(f(x)), x\right) \longrightarrow \pi_{j}(Y, f(x))
$$

where we assume without loss of generality that $f$ has connected fibres.

(2.3.11) Claim. (2.3.10) is an injection for $j \leq k$.

Proof. Let $B$ be the closed unit ball in $R^{j}$ and let $e=(1,0,0, \cdots, 0)$. Let $\gamma:(B, \partial B) \rightarrow\left(X, f^{-1}(f(x))\right)$ be a simplicial map with $\gamma(e)=x$, such that the element $f \circ \gamma$ induces in $\pi_{j}(Y, f(x))$ is trivial. Let $B^{\prime}$ be the closed unit ball in $R^{j+1}$ and let $h: \partial B^{\prime} \rightarrow Y$ be the map induced by $f \circ \gamma$. Let $\tilde{h}: B^{\prime} \rightarrow$ $Y$ with $\left.\tilde{h}\right|_{\partial B^{\prime}}=h$ be a simplicial map which exists by (0.5.1.1) and the fact that $h$ gives rise to a trivial element of $\pi_{j}(Y, f(x))$. By (2.3.9), one pulls $f$ back to get a $k$ quasi-fibration $f^{\prime}: X^{\prime} \rightarrow B^{\prime}$ with $\tilde{h}^{\prime}: X^{\prime} \rightarrow X$ the induced map. Let $x^{\prime} \in X^{\prime}$ be such that $\tilde{h}^{\prime}\left(x^{\prime}\right)=x$. Now $\gamma$ induces a map $\gamma^{\prime}:(B, \partial B)$ $\rightarrow\left(X^{\prime}, f^{-1}\left(f^{\prime}\left(x^{\prime}\right)\right)\right)$. By (2.3.8) the $\operatorname{map} \pi_{j}\left(X^{\prime}, f^{\prime-1}\left(f^{\prime}\left(x^{\prime}\right)\right), x^{\prime}\right) \rightarrow \pi_{j}\left(B^{\prime}, f^{\prime}(x)\right) \approx 0$ is an isomorphism. This implies that $\gamma^{\prime}$ and hence $\gamma$ are trivial elements of $\pi_{j}\left(X^{\prime}, f^{\prime-1}\left(f^{\prime}\left(x^{\prime}\right)\right), x^{\prime}\right)$ and $\pi_{j}\left(X, f^{-1}(f(x)), x\right)$ respectively.

(2.3.12) Claim. (2.3.10) is a surjection $j \leq k+1$.

Proof. Let $B$ be the closed unit ball in $R^{j}$ with $e=(1,0,0, \cdots, 0)$. By (2.3.3) we can assume that $j>1$. Let $\gamma:(B, \partial B) \rightarrow(Y, f(x))$ be a simplicial map representing an element of $\pi_{j}(Y, f(x))$.

Now let $f^{\prime}: X^{\prime} \rightarrow B$ be the pullback of $f$ by $\gamma$. By (2.3.9) it is a $k$ quasi-fibration. Let $h: \partial B \rightarrow X^{\prime}$ be the continuous map such that $f^{\prime} \circ h$ is the identity and $\gamma^{\prime} \circ h(e)=x$ where $\gamma^{\prime}: X^{\prime} \rightarrow X$ is the map induced by $\gamma$.

By (2.3.8) we have the exact sequence:

$$
\pi_{j-1}\left(f^{\prime-1}\left(f^{\prime}\left(x^{\prime}\right)\right), x^{\prime}\right) \longrightarrow \pi_{j-1}\left(X^{\prime}, x^{\prime}\right) \longrightarrow 0 \text {. }
$$

Thus there is a continuous map $H: \partial B \times[0,1] \rightarrow X^{\prime}$ with $\left.H\right|_{\partial B \times 0}=h$, $H(\partial B \times 1) \subseteq f^{\prime-1}\left(f^{\prime}(x)\right)$, and $H(e, t)=x^{\prime}$ for all $t \in[0,1]$. Let $H^{\prime}:(B, \partial B) \rightarrow$ $\left(X, f^{-1}(f(x))\right)$ be the map induced by $\gamma \circ H$. We will be done if we show that $f \circ H^{\prime}$ induces the same element of $\pi_{j}(Y, f(x))$ as $\gamma$. This will follow from (0.5.2) by showing that the map $\tilde{H}: B \rightarrow B$ induced by $f^{\prime} \circ H$ is the identity on $\partial B$. This is clear since $\left.f^{\prime} \circ H\right|_{\partial B \times 0}=f^{\prime} \circ h$ is the identity.

It is not hard to use the above to prove 'generalized Lefschetz' theorems of a type somewhat different than those in $\S 3$. Here we will content ourselves with a simple application that illustrates some of the restrictions 
imposed by $\lambda$ convexity. Recall that a connected complex manifold $W$ is $\lambda$ convex in the sense of Andreotti-Grauert [2] if there is a proper $C^{\infty}$ map $f: W \rightarrow[0, \infty)$ whose Levi form has at most $\lambda-1$ non-positive eigenvalues on the complex tangent space of each point of $W$ outside of a compact set $K \subseteq W$. If $X$ is a compact complex manifold and $A$ is an analytic subspace of $X$, then $X-A$ is $\lambda$ convex if and only if $X-A$ possesses an asymptotically $\lambda$ convex exhaustion function [see 28 , Lemma (0.3.3) for a proof of this].

(2.4) Corollary. Let $A$ be a connected complex submanifold of $\boldsymbol{P}_{\boldsymbol{C}}^{N}$. Let $A^{\prime}$ be the cone on $A$ from a point, $x$, in $\boldsymbol{P}_{C}^{N+1}$ where $\boldsymbol{P}_{C}^{N}$ is assumed to be a linear subspace of $\boldsymbol{P}_{C}^{N+1}$. Then $H^{1}(A, C) \neq 0$ implies $\boldsymbol{P}_{C}^{N+1}-A^{\prime}$ cannot be $\lambda$ convex for $\lambda \leq N-2$.

Proof. Let $\tilde{L}$ denote the $\boldsymbol{P}_{\boldsymbol{C}}^{N}$ in the hypotheses that contains $A$. Then $H^{1}\left(\tilde{L} \cap A^{\prime}, C\right)=H^{1}(A, C) \neq 0$. Further $H^{1}\left(L^{\prime} \cap A^{\prime}, C\right)=0$ where $L^{\prime}$ is any $\boldsymbol{P}_{C}^{N}$ gotten by taking the cone from the $x$ of the hypotheses on a $\boldsymbol{P}_{C}^{N-1}$ inside $\tilde{L}$. But an easy consequence of (2.3) and (1.3) is that as an abstract group, $H^{1}\left(L \cap A^{\prime}, C\right)$ is independent of the hyperplane $L$ of $P_{\boldsymbol{C}}^{N+1}$ if $2 \leq$ $\operatorname{dim}_{C} L-\lambda=N-\lambda$.

\section{§3. The generalized lefschetz theorem}

(3.0) The following, unless otherwise stated, will be standing notation and assumptions throughout this section.

(3.0.1) $X$ is a connected homogeneous complex manifold, i.e. there is a connected Lie group $G$ and a transitive real analytic action $\rho: G \times$ $X \rightarrow X$ where for any $g \in G, \rho(g, x):\{g\} \times X \rightarrow X$ is a biholomorphism. Let $A$ be a connected complex submanifold of $X$ and let $B$ be an analytic subspace of $X$.

(3.0.2) Assume that there exists a $C^{\infty}$ asymptotically $\lambda$ convex exhaustion function $f$ on $U-B$ where $U$ is an open set of $X$ containing $B$.

(3.0.3) Assume all irreducible components of $A$ and $B$ have dimension at least $\lambda$.

(3.0.4) Assume that if $B$ is non-compact, then $B$ is connected and $A$ is compact.

(3.1) I now need some notation and a lemma that do not come into 
the hypotheses of the main results. I therefore suggest that the reader go directly to the statements and proofs of (3.2), (3.3), and (3.4) and come back to (3.1) only as needed.

(3.1.1) For convenience I will often denote $\rho(g, x)$ by $x g$ where $g \in G$ and $x$ is either an element or a subset of $X$. Given a subset $S$ of $X$, denote by $\rho_{S}$ the restriction of $\rho$ to $G \times S$ and by $i_{S}$ the inclusion of $S$ in $X$. Let $\tilde{B}=\{(g, a) \in G \times A \mid a g \in B\}$, and let $\tilde{\mu}: \tilde{B} \rightarrow G \times A$ be the inclusion map. Let $\mu: G \times B \rightarrow G \times X$ be the map $\mu(g, b)=\left(g, b g^{-1}\right)$. Let $\tilde{j}: \tilde{B} \rightarrow G \times B$ denote the map $\tilde{j}(g, a)=(g, a g)$, and let $j: G \times A \rightarrow G \times X$ denote the inclusion map. There is the commutative diagram:

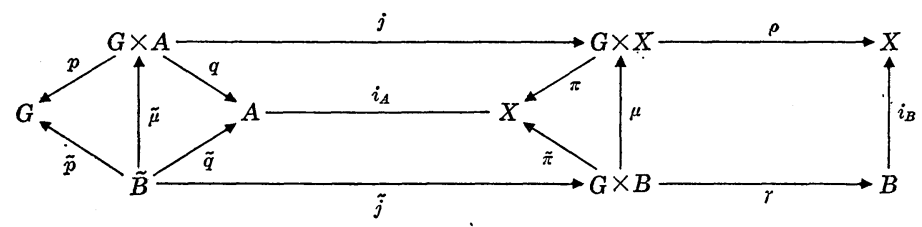

where $p, q, \pi$ and $\gamma$ are product projections, $\tilde{p}=p \circ \tilde{\mu}, \tilde{q}=q \circ \tilde{\mu}$, and $\tilde{\pi}=$ $\pi \circ \mu$.

(3.1.3) Lemma. $\tilde{p}(\tilde{B})=G$ and $\tilde{p}$ is a $\operatorname{dim}_{C} A-\lambda$ quasi-fibration.

Proof. Let $\mathscr{U}=\rho^{-1}(U)$. Note that $\mu(G \times B)=\rho^{-1}(B)$. Define $F(g, x)$ $=f(x g)$ and note that $F\left(x_{n}\right) \rightarrow \infty$ for any sequence $\left\{x_{n}\right\} \subseteq \mathscr{U}-\mu(G \times B)$ that converges to a point in $\mu(G \times B)$. Since either $A$ or $B$ is compact, $\tilde{p}$ is proper. This implies that the hypotheses of $\S 1$ are satisfied with $A, G, \tilde{B}, \tilde{p}, \mathscr{U} \cap(G \times A), \lambda$, and $\left.F\right|_{\mathscr{\ell} \cap(G \times A)}$ equal to $X, S, \mathscr{A}, p, U, \lambda$, and $f$.

By the transitivity of $G, \tilde{B}$ is non-empty and thus $\tilde{p}(\tilde{B})=G$ by (1.1). By (1.2), (1.3), and the definition (2.1) of $k$ quasi-fibration, (3.1.3) is proven.

(3.2) Proposition. $B$ is connected.

Proof. This is part of Proposition (3.1) of [28].

(3.3) Proposition. Given a $d \leq \operatorname{dim}_{C} A-\lambda, \pi_{j}(A, A \cap B, x)=0$ for $x \in A \cap B$ and $j \leq d$ if and only if $\pi_{j}(X, B, b)=0$ for $b \in B$ and $j \leq d$.

Proof. We do this in a sequence of lemmas that move us around (3.1.2). I leave to the reader in each case the check that basepoints are no problem. This check is trivial on noting that $B, A$, and $G$ are connected. 
(3.3.1) Lemma. $\pi_{j}(A, A \cap B, x)=0$ for $x \in A \cap B$ and $j \leq d \leq \operatorname{dim}_{c} A$ $-\lambda$ if and only if $\pi_{j}(G \times A, \tilde{\mu}(\tilde{B}), y)=0$ for $y \in \tilde{\mu}(\tilde{B})$ and $j \leq d \leq \operatorname{dim}_{C} A-\lambda$.

Proof. Using (3.1.3) and (2.3) we have the following commutative diagram with exact rows:

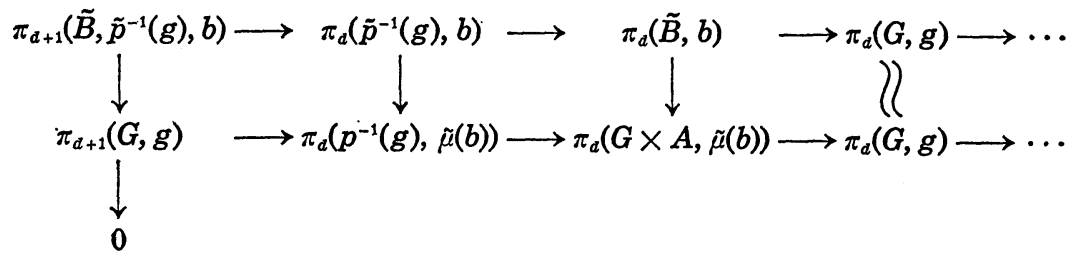

where $g \in G$ and $b \in \tilde{p}^{-1}(g)$, and $d=\operatorname{dim}_{C} A-\lambda$.

Note that $\tilde{p}^{-1}\left(i d_{G}\right)=A \cap B$, and use the five lemma to get (3.3.1).

(3.3.3) Lemma. $\pi_{j}(G \times A, \tilde{\mu}(\tilde{B}), y)=0$ for $j \leq d$ and $y \in \tilde{\mu}(\tilde{B})$ if and only if $\pi_{j}\left(q^{-1}(q(y)), \tilde{\mu}\left(\tilde{q}^{-1}(q(y)), y\right)=0\right.$ for $j \leq d$ and $y \in \tilde{\mu}(\tilde{B})$.

Proof. There is the commutative diagram (cf. (3.1.2))

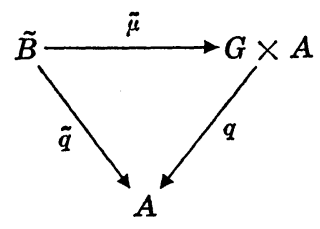

of fiber bundles.

From (3.3.4) we get a commutative diagram of the long exact sequences of homotopy groups associated to $\tilde{q}$ and $q$. Exactly as in the last lemma we prove (3.3.3) by a double use of the five lemma.

Note (cf. (3.1.2)) that (3.3.4) is the pullback of:

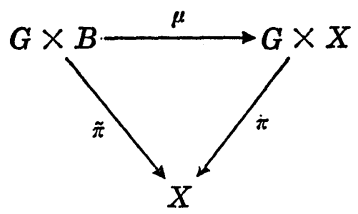

In particular, $j$ gives a homeomorphism of the pairs $\left(q^{-1}(\tilde{q}(x))\right.$, $\left.\tilde{\mu}\left(\tilde{q}^{-1}(\tilde{q}(x))\right)\right)$ and $\left(\pi^{-1}(\tilde{\pi}(\tilde{j}(x))), \mu\left(\tilde{\pi}^{-1}(\tilde{\pi}(\tilde{j}(x)))\right)\right)$ for $x \in \tilde{B}$. This and the same reasoning as in (3.3.3) applied to (3.3.5) gives:

(3.3.6) Lemma. $\pi_{j}\left(q^{-1}(q(y)), \tilde{\mu}\left(\tilde{q}^{-1}(q(y))\right), y\right)=0$ for $j \leq d$ and $y \in \tilde{\mu}(\tilde{B})$ if and only if $\pi_{j}(G \times X, \mu(G \times B), x)=0$ for $j \leq d$ and $x \in \mu(G \times B)$. 
Consider:

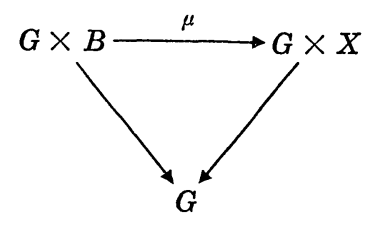

where the vertical maps are the product projections. Note that

$$
\left.\mu\right|_{\left\{\mathrm{id}_{G\} \times B}\right.}:\left\{\mathrm{id}_{G}\right\} \times B \longrightarrow\left\{\mathrm{id}_{G}\right\} \times X
$$

is simply the inclusion. By the same reasoning as (3.3) this gives:

(3.3.8) Lemma. $\pi_{j}(G \times X, \mu(G \times B), x)=0$ for $j \leq d$ and $x \in \mu(G \times B)$ if and only if $\pi_{j}(X, B, b)=0$ for $b \in B$ and $j \leq d$.

Now simply combine (3.3.1), (3.3.3), (3.3.6), and (3.3.8).

(3.4) Proposition. If $\rho(g, A) \subseteq B$ for some $g \in G$, then $\pi_{j}(X, B, b)=0$ for $j \leq \operatorname{dim}_{C} A-\lambda+1$. In particular if $B$ is a compact complex submanifold of $X$, then $\pi_{j}(X, B, b)=0$ for all $b \in B$ and $j \leq \operatorname{dim}_{C} B-\lambda+1$.

Proof. If $\rho(g, A) \subseteq B$ then $\tilde{\mu}$ gives a homeomorphism of $p^{-1}(g)$ with $\tilde{p}^{-1}(g)$.

Now consider the commutative diagram of exact sequences with $d=$ $\operatorname{dim}_{C} A-\lambda$ :

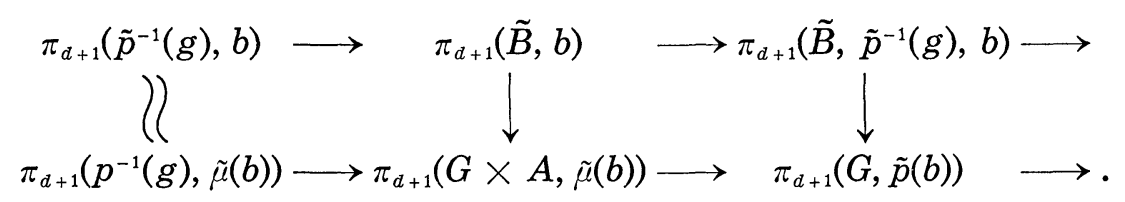

Since $A$ is connected and $\tilde{p}^{-1}(g)$ is homeomorphic to $A$, we have $\pi_{0}\left(\tilde{p}^{-1}(g), b\right) \approx \pi_{0}\left(p^{-1}(g), \tilde{\mu}(b)\right)=0$. We also have from (2.3) that the right hand vertical arrow of (3.4.1) is a surjection. Now note that (3.4.1) can be continued as (3.3.2). Thus by the five lemma $\pi_{j}(G \times A, \tilde{\mu}(\tilde{B}), \tilde{\mu}(b))=0$ for $b \in \tilde{B}$ and $j \leq \operatorname{dim}_{c} A-\lambda+1$. Using the lemmas (3.3.3), (3.3.6), and (3.3.8), we get $\pi_{j}(X, B, b)=0$ for $b \in B$ and $j \leq \operatorname{dim}_{C} A-\lambda+1$. Take $A$ $=B$ if $B$ is a compact complex manifold, in order to finish.

(3.5) Corollary. Let $X, B, \lambda$ etc. be as in (3.0). Let it be assumed that $B$ is projective with singular set $B^{\prime}$. Then

$$
\pi_{j}(X, B, b)=0
$$


for all $b \in B$ and $j \leq \operatorname{dim}_{C} B-\operatorname{dim}_{C} B^{\prime}-\lambda$ where $\operatorname{dim}_{C} B^{\prime}=-1$ if $B^{\prime}$ is empty.

Proof. Let $A$ be a submanifold of $B$ of dimension $\operatorname{dim}_{C} B-\operatorname{dim}_{C} B^{\prime}$ -1 whose intersection with $B^{\prime}$ is empty. This is possible since $B$ is projective. Then by $(3.4), \pi_{j}(X, B, b)=0$ for $j \leq \operatorname{dim}_{c} A-\lambda+1=\operatorname{dim}_{C} B$ $-\operatorname{dim}_{C} B^{\prime}-1-\lambda+1=\operatorname{dim}_{C} B-\operatorname{dim}_{C} B^{\prime}-\lambda$.

(3.6) Proposition. In the above results $\lambda$ can be chosen as:

(3.6.1) $\operatorname{cod}_{C} B$, if $B$ is a manifold and $X$ is a simple Abelian variety,

(3.6.2) $\operatorname{cod}_{c} B$, if $B$ is a compact manifold with ample (in the sense of Hartshorne, i.e., Grauert positive or cohomologically positive in the sense of Griffiths) normal bundle,

(3.6.3) $\operatorname{cod}_{C} B-\min _{i}\left\{n_{i}\right\}+\operatorname{dim}_{C} X$, if $B$ is a reduced local complete intersection with at worst isolated singularities, and $X=\prod_{i} \operatorname{Gr}\left(r_{i}, n_{i}\right)$ where $\operatorname{Gr}\left(r_{i}, n_{i}\right)$ is the Grassmannian of $\boldsymbol{P}_{C}^{r_{i}}$ 's in $\boldsymbol{P}_{\boldsymbol{C}}^{n_{i}}$.

Proof. The above are the main results of [27].

(3.7) Remark. The above results are easily checked to be true if $A$ is replaced by a proper holomorphic immersion $\phi: A \rightarrow X$. In this case we work with the pair $\left(A, \phi^{-1}(B)\right)$ instead of $(A, A \cap B)$.

\section{§4. Closing remarks}

I have recently shown [cf. 29]:

(4.1) Proposition. Let $X=X_{1} \times X_{2}$ where $X_{1}$ is an Abelian variety with $k$ the minimum of the dimensions of the non-zero Abelian varieties of $X_{1}$, and $X_{2}=\prod_{i} \operatorname{Gr}\left(r_{i}, n_{i}\right)$ as in (3.6.3). Then given a pure dimensional complex submanifold $A$ of $X, X-A$ is $\lambda=\operatorname{cod}_{C} A+\operatorname{dim}_{C} X-\min _{i}\left\{k, n_{i}\right\}$ convex.

Thus, by looking over (3.6), we see that the results of this paper and [28] immediately apply to a considerably larger class of manifolds.

The next problem is a very pressing one:

(4.2) Problem. Given an analytic set $B$ in a homogeneous complex manifold $X$, how do the singularities of $B$ affect the choice of the best $f$ and $\lambda$ in (3.0.2). 
The simple result (2.4) shows that the singularities impose interesting and strong 'local' constraints. It would be hoped that one could show that the best $\lambda$ globally allowed for smooth manifolds, would work for $B$ subject only to the restrictions imposed by singularities [cf. 19 and the interesting notion t.a.b.].

Now let me mention a few examples.

(4.3) $\boldsymbol{P}_{\boldsymbol{C}}^{1} \times \boldsymbol{P}_{\boldsymbol{C}}^{2 n+1}$ is a submanifold of $2^{4 n+2}$ the $4 n+2$ dimensional hyperquadric. To see this let $\left\{z_{0}, z_{1}\right\}$ and $\left\{w_{0}, \cdots, w_{2 n+1}\right\}$ be homogeneous coordinates on $\boldsymbol{P}_{C}^{1}$ and $\boldsymbol{P}_{C}^{2 n+1}$ respectively. Let $\mathscr{Y}_{i j}=w_{j} z_{j}$ be the Segre embedding of $\boldsymbol{P}_{\boldsymbol{C}}^{1} \times \boldsymbol{P}_{\boldsymbol{C}}^{2 n+1}$ into $\boldsymbol{P}_{\boldsymbol{C}}^{4 n+3}$. Let $2^{4 n+2}$ be defined by

$$
\sum_{i=0}^{n}\left(\mathscr{Y}_{2 i, 0} \mathscr{Y}_{2 i+1,1}-\mathscr{Y}_{2 i+1,0} \mathscr{Y}_{2 i, 1}\right)
$$

Note that $H^{3}\left(2^{4 n+2}, P_{C}^{1} \times P_{C}^{2 n+1}, Z\right) \neq 0$ for $n \geq 1$. Thus the bound for $A$, a submanifold of $2^{4 n+2}$ :

$$
H^{q}\left(\mathscr{Q}^{4 n+2}, A, Z\right)=0 \quad q \leq 2 \operatorname{dim}_{C} A-(4 n+2)=2
$$

that one gets from Barth's theorem for $2^{4 n+2}$ in $P_{C}^{4 n+3}$, is sharp.

(4.4) Let $\operatorname{Gr}(k, n)$ be the Grassmannian of $\boldsymbol{P}_{C}^{k}$ s in $\boldsymbol{P}_{C}^{n}$. By using the formula in [18] or the last chapter of [9], one deduces that the first $j$ such that $H^{j}(\operatorname{Gr}(k, 1+n), \operatorname{Gr}(k, n), Z)$ is non-zero is $j=2(n-k+1)$. Our result says that it is zero for $j \leq n-2 k$.

(4.5) If $X$ is a simple Abelian variety, then generically, relative to the moduli of $X, H^{1}\left(X, T_{X}^{*}\right) \cap H^{2}(X, Q)=Q$. Thus one can use Proposition (3.6), Corollary (3.4), and the argument [13, cf. also 7] to show in this case that any codimension two submanifold of $X$ with $\operatorname{dim}_{C} X \geq 6$, is defined by the vanishing to the first order of a holomorphic section of a holomorphic rank two vector bundle on $X$. I know no example of a codimension two submanifold of a simple Abelian variety $X$ which is not topologically a complete intersection, though it is easy to produce examples that are not holomorphically complete intersections. Indeed, let

$$
0 \longrightarrow \mathcal{O}_{X} \longrightarrow E \longrightarrow \mathcal{O}_{X} \longrightarrow 0
$$

be a non-trivial extension of the trivial bundle itself. $E$ is irreducible.

Now by tensoring $E$ with a high power of a very ample line bundle, one gets sections whose zero sets are not complete intersections. 
I think that the methods of [21] will lead to some non-trivial noncomplete intersections.

Note added in proof. I would like to call attention to the paper Ampleness and connectedness in complex $G / p$ by $\mathrm{N}$. Goldstein, to appear in Trans. Amer. Math. Soc. In it the $\lambda$ that occur in the bounds of our theorems are computed for all rational homogeneous manifolds. I would also like to call attention to the generalized '2nd Lefschetz theorem' fo N. Goldstein, Math. Ann. 246 (1979), 41-68, that bears the same relation to the 2nd Lefschetz theorem for hyperplane sections as the results in this paper bear to the 1st Lefschetz theorem for hyperplane sections.

\section{BIBLIOGRAPHY}

[1] Andreotti, A. and Frankel, T., The Lefschetz theorem on hyperplane sections, Ann. of Math., 69 (1959), 713-717.

[2] Andreotti, A. and Grauert, H., Théorèmes de finitude pour la cohomologie des espaces complexes, Bull. Soc. Math. France, 90 (1962), 193-259.

[3] Barth, W., Der Abstand von einer algebraischen Mannigfaltigkeit im komplexprojectiven Raum, Math. Annalen, 187 (1970), 150-162.

[4] - Transplanting cohomology classes in complex-projective space, Amer. Jour. of Math., 92 (1970), 951-967.

[5] - Larsen's theorem on the homotopy groups of projective manifolds of small embedding codimension, Proc. Symp. in Pure Math., 29 (1975), 307-313.

[6] Barth, W. and Larsen, M. E., On the homotopy groups of complex projective manifolds, Math. Scand., 30 (1972), 88-94.

[ 7 ] Barth, W. and van de Ven, A., A decomposability criterion for algebraic 2 bundles on projective space, Invent. Math., 25 (1974), 91-106.

[ 8 ] Bott, R., On a theorem of Lefschetz, Mich. Math. J., 6 (1959), 211-216.

[ 9 ] Chern, S. S., Complex Manifolds without Potential Theory, Math. Studies 15, D. Van Nostrand Co., Princeton, 1967.

[10] Dold, A. and Thom, R., Quasifaserungen und unendlich Symmetrische Produkte, Ann. of Math., 67 (1958), 239-273.

[11] Fischer, G., Complex Analytic Geometry, Lecture Notes in Math. 538, Springer Verlag, New York, 1976.

[12] Fritzsche, K., $q$-konvex Restmengen in kompakten komplexen Mannigfaltigkeiten, Math. Annalen, 221 (1976), 251-273.

[13] Grauert, H. and Mülich, G., Vektorbündel vom Rang 2 über dem $n$-dimensional komplex-projektiven Raum, Manuscripta Math., 16 (1975), 75-100.

[14] Griffiths, P. A., Hermitian differential geometry, Chern classes, and positive vector bundles, in: Global Analysis, Papers in honor of K. Kodaira, 185-251, Princeton University Press, 1969.

[15] Hartshorne, R., Cohomology of non-complete algebraic varieties, Compos. Math., 23 (1971), 257-264.

[16] —, Varieties of small codimension in projective space, Bull. Amer. Math. Soc., 80 (1974), 1017-1032.

[17] Hartshorne, R. and Speiser, R., Cohomological dimension in characteristic p, Ann. of Math., (2) 105 (1977), 45-79. 
[18] Hodge, W. V. D. and Pedoe, E., Methods of Algebraic Geometry, Vol. 2, Cambridge Univ. Press, 1952.

[19] Kaup, L. and Weidner, H., Mayer-Vietoris Sequenzen und Lefschetzsätze für mehrfache Hyperflächenschnitte in der Homotopie, Math. Zeit., 142 (1975), 243269.

[20] Lefschetz, S., L'Analysis sítus et la géométrie algébrique, Selected Papers by S. Lefschetz, Chelsea Publishing Company, New York, 1971.

[21] Matsushima, Y., Heisenberg groups and holomorphic vector bundles over a complex torus, Nagoya Math. J., 61 (1976), 161-195.

[22] Milnor, J., Morse Theory, Princeton University Press, 1963, Second printing, 1965.

[23] Ogus, A., Local cohomological dimension of algebraic varieties, Ann. Math., 98 (1973), 327-365.

[24] Sommese, A. J., On manifolds that cannot be ample divisors, Math. Ann., 221 (1976), 55-72.

[25] — Theorems of Barth-Lefschetz type for complex subspaces of homogeneous complex manifolds, Proc. Nat. Acad. Sci. USA, 74 (1977), 1332-1333.

[26] — Submanifolds of Abelian varieties, Math. Ann., 233, (1978), 229-256.

[27] — Concavity theorems, Math. Ann., 235 (1978), 37-53.

[28] — Complex Subspaces of Homogeneous Complex Manifolds, I-Transplanting Theorems, Duke Jour. of Math., 46 (1979), 527-548.

[29] — A convexity theorem, to appear in Proc. Symp. in Pure Math. on Singularities, Arcata, 1981.

[30] Spanier, E. H., Algebraic Topology, New York, McGraw-Hill, 1966.

Department of Mathematics

University of Notre Dame

Notre Dame, Indiana, 46556

U.S.A. 